\title{
Clusterin ameliorates tau pathology in vivo by inhibiting fibril formation
}

\author{
Aleksandra M. Wojtas 1,3, Yari Carlomagno², Jonathon P. Sens ${ }^{1,3}$, Silvia S. Kang², Tanner D. Jensen', \\ Aishe Kurti ${ }^{1}$, Kelsey E. Baker ${ }^{1}$, Taylor J. Berry', Virginia R. Phillips², Monica Casey Castanedes², Ayesha Awan', \\ Michael DeTure², Cristhoper H. Fernandez De Castro², Ariston L. Librero², Mei Yue², Lillian Daughrity², \\ Karen R. Jansen-West ${ }^{2}$, Casey N. Cook ${ }^{2,3}$, Dennis W. Dickson ${ }^{2,3}$, Leonard Petrucelli ${ }^{2,3}$ and John D. Fryer ${ }^{1,3^{*}}$
}

\begin{abstract}
The molecular chaperone Clusterin (CLU) impacts the amyloid pathway in Alzheimer's disease (AD) but its role in tau pathology is unknown. We observed CLU co-localization with tau aggregates in AD and primary tauopathies and CLU levels were upregulated in response to tau accumulation. To further elucidate the effect of CLU on tau pathology, we utilized a gene delivery approach in CLU knock-out (CLU KO) mice to drive expression of tau bearing the P301L mutation. We found that loss of CLU was associated with exacerbated tau pathology and anxiety-like behaviors in our mouse model of tauopathy. Additionally, we found that CLU dramatically inhibited tau fibrilization using an in vitro assay. Together, these results demonstrate that CLU plays a major role in both amyloid and tau pathologies in AD.
\end{abstract}

Keywords: Clusterin, Alzheimer's disease, Tauopathy, Tau

\section{Introduction}

Alzheimer's disease (AD) is a highly prevalent neurodegenerative disorder characterized by deposition of amyloid- $\beta$ (A $\beta$ ) peptide as parenchymal plaques [7] and aggregation of hyperphosphorylated protein tau as neurofibrillary tangles (NFTs) [8]. Aberrant tau accumulation is also a pathological hallmark of other neurodegenerative disorders, collectively known as primary tauopathies, including progressive supranuclear palsy (PSP), corticobasal degeneration (CBD), Pick's disease (PiD), and frontotemporal dementia with parkinsonisms linked to chromosome 17 (FTDP-17), in which amyloid pathology is not usually observed [23]. Human tauopathies are often classified based on the presence of different tau isoforms that contain three or four carboxy-terminal

\footnotetext{
*Correspondence: fryer.john@mayo.edu

1 Department of Neuroscience, Mayo Clinic, Collaborative Research Building CR03-010 13400 E. Shea Blvd, Scottsdale, AZ 85259, USA

Full list of author information is available at the end of the article
}

repeat domains in the tau aggregates, including 3R (PiD), 4R (PSP, CBD), and 3R + 4R (AD) [12, 23]. Given that tau accumulation strongly correlates with neuronal dysfunction and cognitive impairment in $\mathrm{AD}$ and other tauopathies $[1,11]$, identifying factors that regulate tau aggregation in the brain is critical.

Compelling evidence implicates the clusterin $(C L U)$ gene in $\mathrm{AD}$ pathophysiology. Several large-scale genomewide association studies (GWAS) have identified a number of single nucleotide polymorphisms (SNPs) at the $C L U$ locus that are significantly associated with altered $\mathrm{AD}$ risk $[9,13]$. In addition, levels of CLU (also known as apolipoprotein J, apoJ) have been found to be increased in AD-affected brain regions [15] and CLU is highly elevated in the cerebrospinal fluid [16] and plasma of AD patients [19]. Previous studies have shown that CLU binds to $A \beta$ affecting its deposition [4, 24], fibrillogenesis [17], and clearance [2, 27], thus suggesting that CLU is a key regulator of $A \beta$ metabolism. Moreover, CLU involvement in a plethora of physiological and pathological processes have been attributed to its prominent original author(s) and the source, provide a link to the Creative Commons licence, and indicate if changes were made. The images or other third party material in this article are included in the article's Creative Commons licence, unless indicated otherwise in a credit line to the material. If material is not included in the article's Creative Commons licence and your intended use is not permitted by statutory regulation or exceeds the permitted use, you will need to obtain permission directly from the copyright holder. To view a copy of this licence, visit http://creativecommons.org/licenses/by/4.0/. The Creative Commons Public Domain Dedication waiver (http://creativeco mmons.org/publicdomain/zero/1.0/) applies to the data made available in this article, unless otherwise stated in a credit line to the data. 
role as a major extracellular chaperone [5]. However, it is unknown whether CLU affects AD risk by modulating tau pathology independently of $A \beta$.

\section{Materials and methods Animals}

6-Month-old C57BL/6J (CLU wild-type, CLU WT) and CLU knock-out (CLU KO) mice were used in this study. Mice were housed in a temperature and humidity-controlled environment under a 12-h light/dark cycle and with free access to food and water. All studies were performed in accordance with National Institute of Health Guide for the Care and Use of Laboratory Animals (National Research Council (2011) Guide for the Care and Use of Laboratory Animals (National Academies Press, Washington, DC), 8th Ed.) under the approved protocol from the Mayo Clinic Institutional Animal Case and Use Committee. Both male and female mice were included in the study.

\section{Histological and biochemical evaluation of human postmortem tissue}

To examine CLU association with tau, human postmortem brain tissues were obtained from Mayo Clinic Jacksonville Brain Bank from age-matched individuals diagnosed with primary and secondary tauopathies, including $\mathrm{CBD}, \mathrm{PiD}, \mathrm{AD}$, and non-demented controls (Additional file 1: Table S1). For histological analyses, paraffin-embedded hippocampal sections were cut 5 $\mu \mathrm{m}$, then deparaffinized using xylene and rehydrated in a graded series of alcohols. Antigen retrieval was achieved by incubating tissue sections in distilled water for 30 min under high temperature. Immunostaining was performed on the Thermo Scientific Autostainer 480S, using the Envision G/2 doublestain kit (Dako, Carpinteria, CA). Peroxidase (HRP) labeling of rabbit polyclonal Clusterin antibody (1:2500, Abcam, Cambridge, MA) was visualized using $3,3^{\prime}$-diaminobenzidine $(\mathrm{DAB}+)$ as the chromogen. Alkaline phosphatase labeling of mouse monoclonal antibody MC1 (1:250, gift from Peter Davies, Feinstein Institute for Medical Research), was visualized using Vector Blue Alkaline Phosphatase Substrate kit (Vector Laboratories, Burlingame, CA). The sections were then dehydrated, coverslipped without counterstaining using permanent mounting media, scanned using the Aperio Slide Scanner (Aperio, Vista, CA), and analyzed using the ImageScope software (Aperio, Vista, CA).

Human brain sections were also incubated overnight with goat anti-CLU antibody (1:50, sc-6420, Santa Cruz Biotechnology) followed by double labeling with thioflavine-S to detect mature tau tangles. The slides were then incubated with Sudan black B (Sigma S0395) for $30 \mathrm{~min}$ at RT in order to eliminate lipofuscin-like autofluorescence. The images were captured using a Zeiss LSM 880 confocal microscope.

For biochemical analysis, frontal cortex from tauopathy cases and non-demented individuals was used. Demographic and neuropathological features of human subjects are presented in Additional file 1: Table S2. TBS buffer (50 mM Tris, pH8.0, $274 \mathrm{mM}$ $\mathrm{NaCl}, 5 \mathrm{mM} \mathrm{KCl}, 1 \mathrm{mM}$ PMSF, and protease and phosphatase inhibitor cocktail) was added to cortex and manually homogenized using glass dounce homogenizator. The homogenates were then ultracentrifuged at $150,000 \times g$ for $15 \mathrm{~min}$ at $4{ }^{\circ} \mathrm{C}$ and the supernatant (S1 fraction) was transferred to a new Eppendorf tube. The total CLU levels in the S1 fraction from human tissues were measured using the Human Clusterin DuoSet enzyme-linked immunosorbent assay (ELISA) (R\&D Systems, DY5874) according to the manufacturer's instructions. Total protein concentrations used for normalization were assessed by Bicinchoninic Acid (BCA) Protein Assay kit (Thermo Scientific), according to the manufacturer's instructions with a standard curve using BSA.

\section{AAV-GFP and AAV-Tau ${ }^{\text {P301L }}$ viral production}

Viral vector construction and AAV9 production was performed, as previously described [3]. Briefly, V5-tagged $\mathrm{Tau}^{\mathrm{P} 301 \mathrm{~L}}$ or GFP expression plasmids were cloned into an AAV vector. The constructs were sequence-verified using ABI3730 with Big Dye chemistry (Applied Biosystems, Foster City, CA). AAV vectors expressing GFP and $\mathrm{Tau}^{\mathrm{P} 301 \mathrm{~L}}$ under the control of the cytomegalovirus enhancer/chicken $\beta$-actin promoter, as well as a woodchuck post-transcriptional regulatory element and the bovine growth hormone polyA, were co-transfected with AAV helper plasmids into HEK293T cells. Cells were harvested and lysed in the presence of $0.5 \%$ sodium deoxycholate and $50 \mathrm{U} / \mathrm{ml}$ Benzonase (Sigma, St. Louis, MO) by freeze thawing $48 \mathrm{~h}$ post-transfection, and the virus was isolated using a discontinuous iodixanol gradient. Quantitative PCR was used to measure the genomic titer of each virus.

\section{Intracerebroventricular injections}

AAV9-GFP or AAV9-Tau ${ }^{\text {P301L }}$ viruses were injected bilaterally into cerebral lateral ventricles of C57BL/6 J (CLU WT) and CLU KO pups at postnatal day 0 with $2 \mathrm{E}+10$ viral particles/ventricle. Briefly, newborn pups were cryoanasthesized and placed on a cold metal plate followed by piercing the skull with the 30 -gauge needle just posterior to Bregman and $2 \mathrm{~mm}$ lateral to the midline. $2 \mu \mathrm{l}$ of AAV virus was injected into the each lateral ventricle. Following the injections, pups were placed on the 
warm pad until they regained normal color and resumed movement.

\section{Behavioral assessment}

The behavioral analysis consisting of open field test (OFA), elevated plus maze (EPM), and contextual and cued fear conditioning (CFC) was performed on 6-month-old CLU WT and CLU KO mice injected with AAV-GFP or AAV-Tau ${ }^{\text {P301L }}$. Mice were acclimated to the testing room for $1 \mathrm{~h}$. All behavioral equipment was extensively cleaned with $30 \%$ ethanol between each animal. After each test animals were returned to their home cages. In the OFA test, that is performed to assess locomotor activity and anxiety, mice were placed in the center of the open-field arena $(40 \times 40 \times 30 \mathrm{~cm}$, $W \times L \times H)$ and allowed to roam freely for $15 \mathrm{~min}$. An overhead camera was used to track movement with AnyMaze software (Stoelting Co., Wood Dale, IL), and mice were analyzed for multiple measures, including total distance traveled, average speed, time mobile, and distance traveled in an imaginary 'center' zone $(14 \times 14 \mathrm{~cm})$. The $E P M$ test is conducted to test for anxiety and the entire maze is elevated $50 \mathrm{~cm}$ from the floor and consists of four arms $(50 \times 10 \mathrm{~cm})$ with two of the arms enclosed with roofless gray walls $(35 \times 15 \mathrm{~cm}, L \times H)$. Mice were tested by placing them in the center of the maze facing an open arm, and their behavior was tracked for 5 min with an overhead camera and AnyMaze software. The CFC test, which is used to assess hippocampal and amygdala associative learning and memory, was conducted in a sound attenuating chamber with a grid floor capable of delivering an electric shock, and freezing was measured with an overhead camera and FreezeFrame software (Actimetrics, Wilmette, IL). Mice were initially placed into the chamber undisturbed for $2 \mathrm{~min}$, during which time baseline freezing behavior was recorded. An 80-dB white noise served as the conditioned stimulus (CS) and was presented for $30 \mathrm{~s}$. During the final $2 \mathrm{~s}$ of this noise, mice received a mild foot shock $(0.5 \mathrm{~mA})$, which served as the unconditioned stimulus (US). After $1 \mathrm{~min}$, another CS-US pair was presented. The mice were removed $30 \mathrm{~s}$ after the second CS-US pair and returned to its home cage. Twenty-four hours later, each mouse was returned to the test chamber and freezing behavior was recorded for $5 \mathrm{~min}$ (context test). Mice were returned to their home cage and placed in a different room than previously tested in reduced lighting conditions for a period of no less than $1 \mathrm{~h}$. For the auditory CS test, environmental and contextual cues were changed, as previously described [3]. The animals were placed in the apparatus for $3 \mathrm{~min}$ and the auditory CS was presented and freezing was recorded for another $3 \mathrm{~min}$ (cued test). Baseline freezing behavior obtained during training was subtracted from the context or cued test to control for animal variability.

\section{Histological analyses}

Following behavioral tests, 6-month-old mice were deeply anesthetized with pentobarbital ( $100 \mathrm{mg} / \mathrm{kg}$ i.p.) and transcardially perfused with phosphate buffered saline (PBS) to remove blood from cerebrovasculature. After brain removal, one hemibrain was drop-fixed in $10 \%$ neutral buffered formalin (Fisher Scientific, Waltham, MA) overnight at $4{ }^{\circ} \mathrm{C}$ followed by switching the brain to $\mathrm{PBS}$ and stored at $4{ }^{\circ} \mathrm{C}$ until histological evaluation. Cortex and hippocampus from the other hemibrain were isolated, frozen on dry ice and stored at $-80{ }^{\circ} \mathrm{C}$ until further processing. Histology was performed on the formalin-fixed hemibrains from CLU WT and CLU KO animals injected with AAV-GFP or AAV-Tau ${ }^{\text {P301L }}$ that were embedded in paraffin, sectioned in a sagittal plane at a thickness of $5 \mu \mathrm{m}$, and mounted on a glass slide. The sections were deparaffinized, rehydrated, and subjected to antigen-retrieval, followed by blocking endogenous peroxidase activity using $0.03 \%$ hydrogen peroxide for $30 \mathrm{~min}$ at RT. To investigate CLU association with tau deposits brain tissues from CLU WT animals injected with AAV-GFP or AAV-Tau ${ }^{\text {P301L }}$ were incubated overnight with goat anti-CLU antibody (1:50, sc-6420 Santa Cruz Biotechnology) and double labeled with thioflavine-S. Images were obtained on a Zeiss LSM 880 confocal microscope. For immunohistochemistry, brain sections were immunostained using the DAKO Autostainer (DAKO North America, Carpinteria, CA) and the DAKO EnVision + HRP system. The CP13 (pS202, 1:1000), PHF-1 (pS396/S404, 1:1500), and MC-1 (conformational epitope, 1:500) antibodies, kindly provided by Dr. Peter Davies (Feinstein Institute for Medical Research, North Shore LIJ Health Care System), and the anti-GFAP (1:2500 Cell Signaling Technology, 3670 Danvers, MA) and anti-IBA1 (1:3000, Wako Chemicals, 019-19741, Richmond, VA) antibodies were used in the study. Gallyas silver stain was performed on a subset of CLU WT and CLU KO animals injected with AAV-GFP or AAV-Tau ${ }^{\text {P301L }}$, as previously described [3]. Following immunohistochemistry, the slides were dehydrated and scanned using the Aperio Slide Scanner (Aperio, Vista, CA). The blinded quantification was performed using the ImageScope software (Aperio, Vista, CA). The analysis of annotated cortical and hippocampal regions was performed using a custom-designed color deconvolution algorithm, as previously described [10]. More specifically, the algorithm was used to detect and measure the optical density of the brown chromagen (tau) and 
the result was presented as a percentage of tau burden within an annotated region.

To measure the hippocampal area three sections for each animal were used. The quantification of hippocampal area was performed using the ImageScope software (Aperio, Vista, CA).

\section{Biochemical analyses}

For biochemical analysis, sequential extraction of tau was performed, as previously described [3]. Briefly, $500 \mu \mathrm{L}$ and $275 \mu \mathrm{L}$ of TBS buffer (50 mM Tris, pH8.0, $274 \mathrm{mM} \mathrm{NaCl}$, $5 \mathrm{mM} \mathrm{KCl}, 1 \mathrm{mM}$ PMSF, and protease and phosphatase inhibitor cocktail) were added to cortex and hippocampus, respectively and homogenized using the manual pestles. For RNA extraction, $75 \mu \mathrm{L}$ of each homogenate was transferred to a new Eppendorf tube and stored in $-80{ }^{\circ} \mathrm{C}$ until further processing. The remaining homogenates were ultracentrifuged at $150,000 \times g$ for $15 \mathrm{~min}$ at $4{ }^{\circ} \mathrm{C}$ and the supernatant (S1 fraction) was transferred to a new Eppendorf tube. The pellet was homogenized in $3 \times$ volume buffer in a high salt buffer $(10 \mathrm{mM}$ Tris, $\mathrm{pH}$ 7.4, $0.8 \mathrm{M} \mathrm{NaCl}, 10 \%$ sucrose, $1 \mathrm{mM}$ EGTA, $1 \mathrm{mM}$ PMSF and protease and phosphatase inhibitor cocktail) and ultracentrifuged at $150,000 \times g$ for $15 \mathrm{~min}$ at $4{ }^{\circ} \mathrm{C}$. The pellet was discarded and the supernatant was transferred to a new Eppendorf tube and incubated with sarkosyl (final concentration of $1 \%$ ) for $1 \mathrm{~h}$ at $37{ }^{\circ} \mathrm{C}$. The incubation was followed by ultracentrifugation $150,000 \times g$ for $1 \mathrm{~h}$ at $4{ }^{\circ} \mathrm{C}$. The supernatant was transferred to a new Eppendorf tube (S2 fraction) and the pellet was resuspended in the TE buffer (10 mM Tris, pH 8.0, 1 mM EDTA) (P3 fraction). All three fractions were stored in $-80{ }^{\circ} \mathrm{C}$ until further processing. Mouse CLU levels were measured using the Mouse Clusterin DuoSet ELISA (R\&D Systems, DY2747), according to manufacturer's instructions. To measure the total tau levels in the S1 and S2 fractions the MesoScale Discovery (MSD) was performed. Human tau specific E1 antibody (1:500) and biotinylated-HT7 antibody (1:250) were used as the capture and detection antibody, respectively. Tau levels were normalized to the total protein levels, measured by the Bicinchoninic Acid (BCA) Protein Assay kit (Thermo Scientific), according to the manufacturer's instructions with a standard curve using BSA.

To examine total tau levels by immunoblotting, soluble fractions (S1) from cortical region of 6-month-old CLU WT-Tau ${ }^{\mathrm{P} 301 \mathrm{~L}}$ and CLU KO-Tau ${ }^{\mathrm{P} 301 \mathrm{~L}}$ mice were used. BCA assay was used to evaluate protein concentrations. Twenty micrograms from each sample was run on AnykD Criterion Protein Gels (BioRad) and transferred to PVDF membrane (Millipore). Membranes were blocked in $5 \%$ non-fat dry milk in TBS with $0.1 \%$
Triton-X-100 (TBST) for $1 \mathrm{~h}$ and incubated overnight at $4{ }^{\circ} \mathrm{C}$ in primary antibodies (anti-E1 1:2000 and antiPHF-1 1:500) diluted in 5\% milk in TBST. Membranes were then incubated in HRP-conjugated secondary antibodies (1:5000; Jackson ImmunoResearch) for $1 \mathrm{~h}$ at room temperature, and detected by ECL (Thermo Fisher Scientific, Rockford, IL). Bands were quantified by analyzing pixel density, and protein levels were normalized to the protein loading control (GAPDH).

\section{Tau filament assembly}

Tau filament assembly was performed with $5 \mu \mathrm{M} 4 \mathrm{R} 0 \mathrm{~N}$ isoform in assembly buffer (10 mMHEPES (pH7.4), $33 \mathrm{mM} \mathrm{NaCl}, 1 \mathrm{mM} \mathrm{MgCl} 2,1.5 \mathrm{mM}$ EGTA, $60 \mu \mathrm{M}$ EDTA, 5uM Thioflavin-T and $20 \mathrm{ug} / \mathrm{ml}$ Heparin) with and without $4 \mu \mathrm{M}$ recombinant CLU (R\&D \#2937-HS050). Reactions were incubated at $37{ }^{\circ} \mathrm{C}$ and shaken in double orbital at $400 \mathrm{rpm}$ on a BMG Fluostar platereader. Thioflavin- $\mathrm{T}$ fluorescence was measured every $30 \mathrm{~min}(450 \pm 10 \mathrm{~nm}$ excitation, $480 \pm 10 \mathrm{~nm}$ emission, bottom read). One silica bead $(800 \mu \mathrm{m}$, Ops diagnostics) was added to each well in a 384 well plate format.

Following the polymerization reaction, samples were ultracentrifuged at $150,000 \times g$ for $40 \mathrm{~min}$ at $4{ }^{\circ} \mathrm{C}$ in a TLA110 rotor at $60,000 \mathrm{rpm}$ to separate soluble and insoluble, aggregated tau. Pellets containing aggregated tau (insoluble fraction) were resuspended in $60 \mu \mathrm{L} 1 \times$ SDS-PAGE sample buffer (Invitrogen), and $10 \mu \mathrm{L} 6 \times$ SDS-PAGE sample buffer was added to $50 \mathrm{uL}$ of the supernatant (soluble fraction). Samples were separated by SDS-PAGE in Tris-glycine gels (Life Technologies), and transferred to PVDF membranes (Millipore). Membranes were blocked in 5\% non-fat dry milk in TBS/0.1\% Triton X-100, and incubated in primary antibody (E1 or biotinylated CLU). Membranes were then incubated in HRP-conjugated secondary antibodies for $1 \mathrm{~h}$ at RT and detected by ECL (Thermo Fisher Scientific, Rockford, IL).

Heparin-free tau assembly assay was also performed with $5 \mu \mathrm{M} 4 \mathrm{R} 0 \mathrm{~N}$, tau PHF seeds and increasing concentrations of recombinant CLU $(0.25 \mu \mathrm{M}-2 \mu \mathrm{M})$. To purify tau PHFs, $150 \mathrm{mg}$ of cortical region from human $\mathrm{AD}$ cases was used for the sequential extraction, as described above. The final $\mathrm{P} 3$ pellets were resuspended in $75 \mu \mathrm{L}$ of TE buffer and $2 \mu \mathrm{L}$ of $\mathrm{P} 3$ fraction was used in the tau assembly assay.

\section{Statistical analyses}

Statistical significance of experiments involving two groups was assessed by Student's $t$ test. The analysis 
of three or more groups were performed by using one-way ANOVA. The behavioral analysis was performed by using two-way ANOVA with Fisher's LSD test. Data present as mean \pm S.E.M. For all statistical analyses GraphPad Prism software was used (GraphPad).

\section{Results}

$\mathrm{CLU}$ is present in tau aggregates and upregulated in human tauopathies

To determine the effect of CLU expression on tau deposition in the brain, we first evaluated the pattern of CLU localization in human primary and secondary

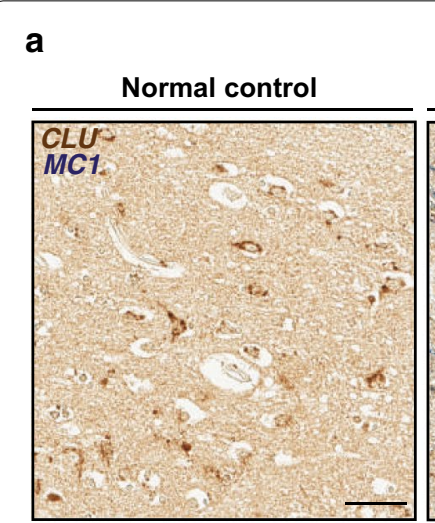

Corticobasal degeneration, CBD (4R tauopathy)

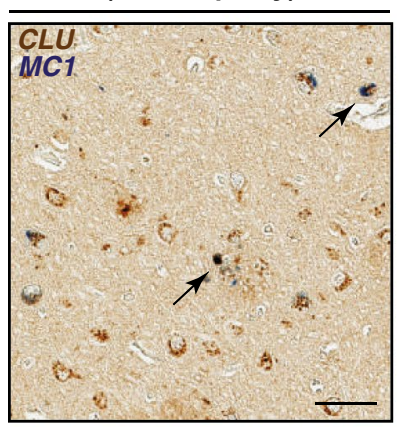

C

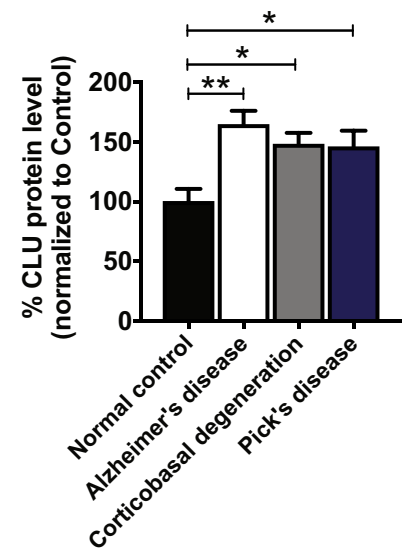

Alzheimer's disease, AD

(3R+4R tauopathy)

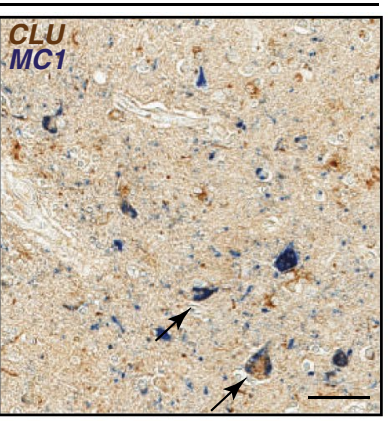

Pick's disease, PiD (3R tauopathy)

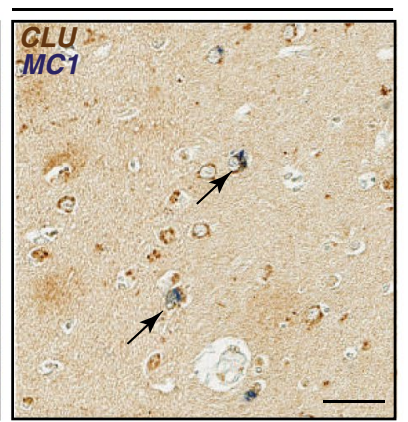

b
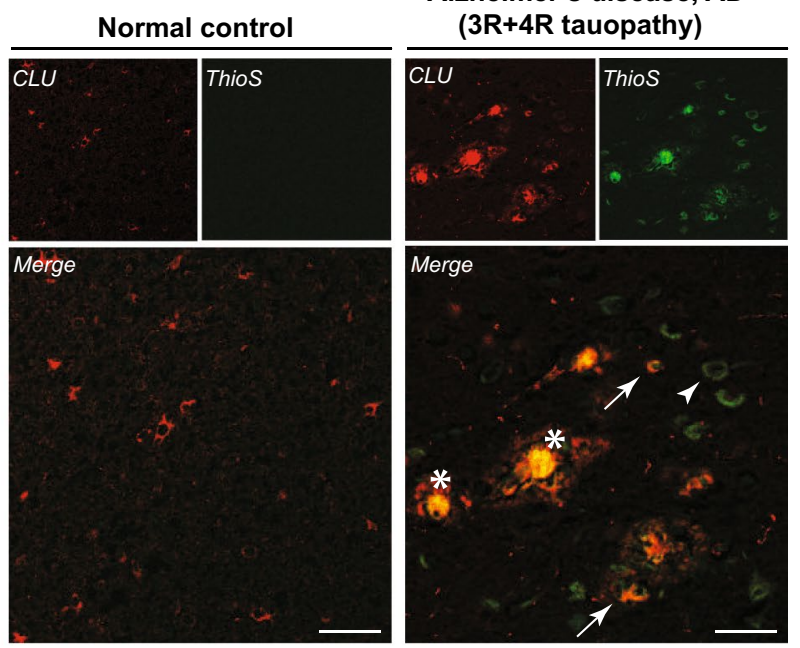

Corticobasal degeneration, CBD (4R tauopathy)
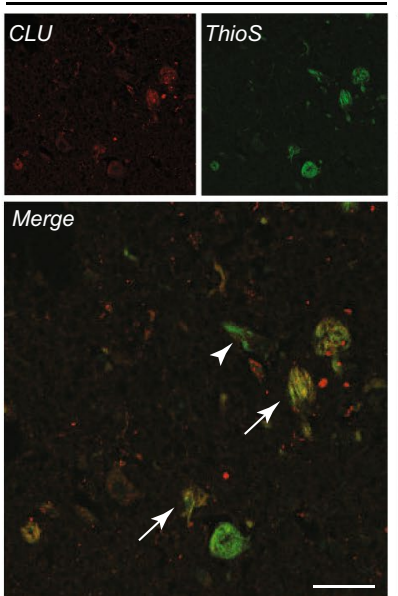

Alzheimer's disease, $A D$

(3R+4R tauopathy)

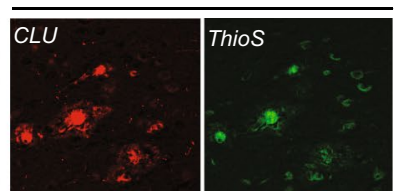

Pick's disease, PiD (3R tauopathy)
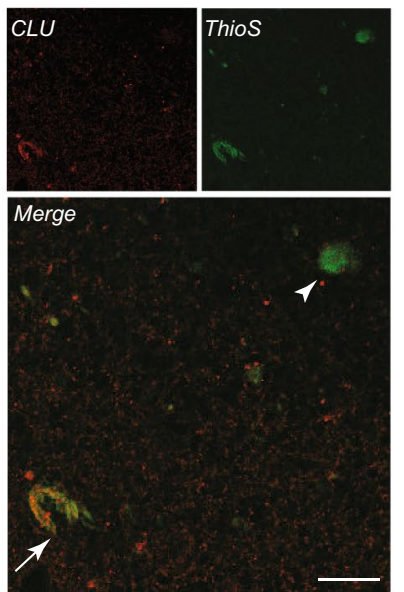

Fig. 1 Clusterin co-localizes with tau deposits and is upregulated in human tauopathies. a Human brain tissues representing normal control, Alzheimer's disease (AD), and the primary tauopathies Pick's disease (PiD) and corticobasal degeneration (CBD). Co-localization of CLU (brown) with tau deposits, marked by MC-1 labeling (blue). Scale bar, $100 \mu \mathrm{m}$. b Arrows indicate co-localization of CLU (red) with mature tau tangles labeled by thioflavine-S staining (green). Arrowheads show tau tangles without CLU co-localization. Asterisks represent amyloid plaques. Scale bar, 100 um. c Biochemical evaluation of the total CLU protein levels in the cortical region of human tauopathies. N=10-15 cases/group. Data presented as mean \pm S.E.M. and analyzed with one-way ANOVA with Tukey's multiple comparison test, ${ }^{*} p<0.05,{ }^{* *} p<0.01$ 
tauopathies. Histological analyses of post-mortem brain tissues from individuals diagnosed with $\mathrm{AD}, \mathrm{CBD}$, and PiD (Additional file 1: Table S1) showed the association of CLU with MC-1-positive tau deposits (Fig. 1a). In addition, CLU co-localized with mature tau tangles, marked by thioflavine-S stain, suggesting the physiological interaction of CLU and tau in vivo (Fig. 1b). We further explored the link between CLU and tau pathology by assessing CLU protein levels in human tauopathies (Additional file 1: Table S2) with a quantitative enzymelinked immunosorbent assay (ELISA). Consistent with previous reports, CLU was highly elevated in AD cases compared to normal controls, an effect partially attributed to the presence of abundant amyloid pathology in AD brains. Notably, we also found a significant increase in CLU levels in primary tauopathies with no amyloid pathology, including $\mathrm{PiD}$ and CBD compared to controls, indicating CLU upregulation in response to tau aggregation (Fig. 1c).

To further investigate the association of CLU with tau accumulation, we used a well-established mouse model of tauopathy [3] in which adeno-associated virus expressing human tau protein bearing the P301L mutation $\left(\mathrm{AAV}-\mathrm{Tau}^{\mathrm{P} 301 \mathrm{~L}}\right)$ or green fluorescent protein as control (AAV-GFP) was delivered to CLU wild-type (WT) and CLU knock-out (KO) animals by intracerebroventricular injections at postnatal day 0 . Consistent with our findings in human brains, CLU co-localized with tau aggregates (Additional file 1: Fig. S1a) and CLU levels were higher in CLU WT-Tau ${ }^{\mathrm{P} 301 \mathrm{~L}}$ mice compared to control animals (Additional file 1: Fig. S1b).
The absence of CLU leads to behavioral abnormalities Given that cognitive and behavioral impairment correlates with tau accumulation in $\mathrm{AD}$ patients and is also present in other tauopathies [1, 11], we assessed the effect of differential CLU expression on anxiety-like behavior as well as learning and memory functions. We did not observe significant differences between CLU genotypes in the total distance traveled (Fig. 2a). However, CLU KO-Tau ${ }^{\mathrm{P} 301 \mathrm{~L}}$ mice had increased anxiety-like behavior, measured by the time spent in a center of the open field arena, compared to CLU WT-Tau ${ }^{\text {P301L }}$ (Fig. 2a). In addition, CLU KO-Tau ${ }^{\text {P301L }}$ mice spent significantly more time in the open arms of the elevated plus maze (EPM) test compared to CLU WT-Tau ${ }^{\mathrm{P} 301 \mathrm{~L}}$ mice, an indication of aberrant exploratory behavior (Fig. 2b). CLU loss did not influence associative learning and memory assessed by the contextual and cued fear conditioning (CFC) tests (Additional file 1: Fig. S2). Overall, these data suggest a significant effect of CLU on anxiety-like behavior in the context of tau pathology.

\section{CLU loss exacerbates tau pathology in a mouse model of tauopathy}

Given that tau is abnormally hyperphosphorylated in AD and other tauopathies [23], we next evaluated the pattern of phosphorylation at different epitopes in cortex and hippocampus of CLU WT-Tau ${ }^{\mathrm{P} 301 \mathrm{~L}}$ and CLU KO$\mathrm{Tau}^{\mathrm{P} 301 \mathrm{~L}}$ animals. CLU KO-Tau ${ }^{\mathrm{P} 301 \mathrm{~L}}$ mice had a modest increase in tau phosphorylation at serine $202(\mathrm{pS} 202)$ detected by CP-13 immunolabeling in cortex $(p=0.068)$ and hippocampus $(p=0.169)$ compared to CLU WT$\mathrm{Tau}^{\mathrm{P} 301 \mathrm{~L}}$ animals (Fig. 3a, b). Since CP-13 detects an

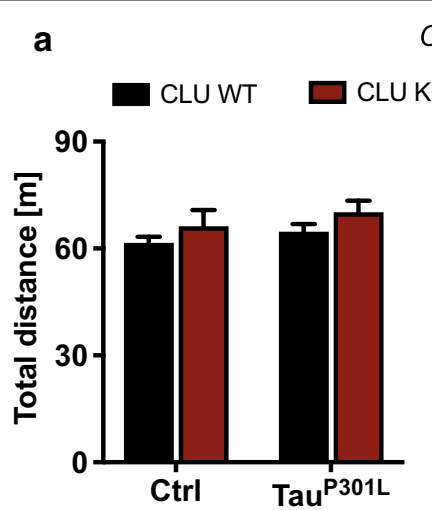

Open field test

b Elevated plus maze
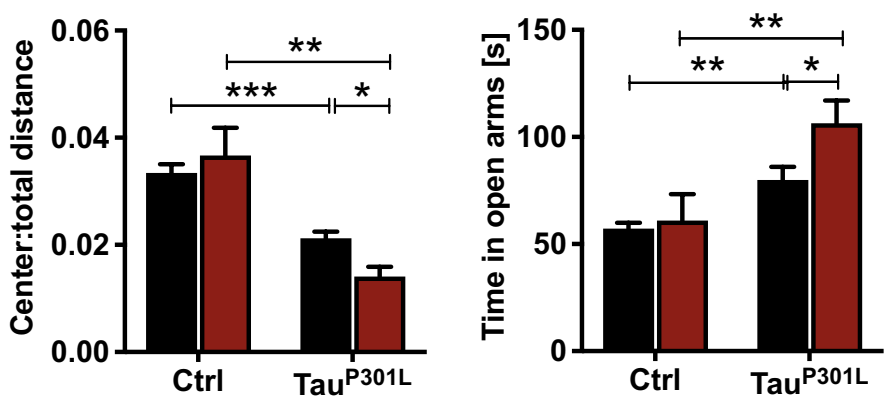

Fig. 2 Loss of Clusterin leads to behavioral abnormalities. a-b Behavioral and cognitive abilities were assessed using a the open field test (OFA) and b elevated plus maze (EPM) in CLU WT-GFP ( $N=49)$, CLU KO-GFP $(N=12)$, CLU WT-TauP301L $(N=65)$, and CLU KO-Tau ${ }^{\text {P301L }}$ ( $N=23$ ). OFA was used to test anxiety-related behavior, evaluated by time spent in the center compared to total time travelled. EPM measured the time spent in the open arms during the test which is a reflection of exploratory behavior. Data present as mean \pm S.E.M. and analyzed with two-way ANOVA with Fisher's LSD test, ${ }^{*} p<0.05,{ }^{* *} p<0.01,{ }^{* * *} p<0.001$ 


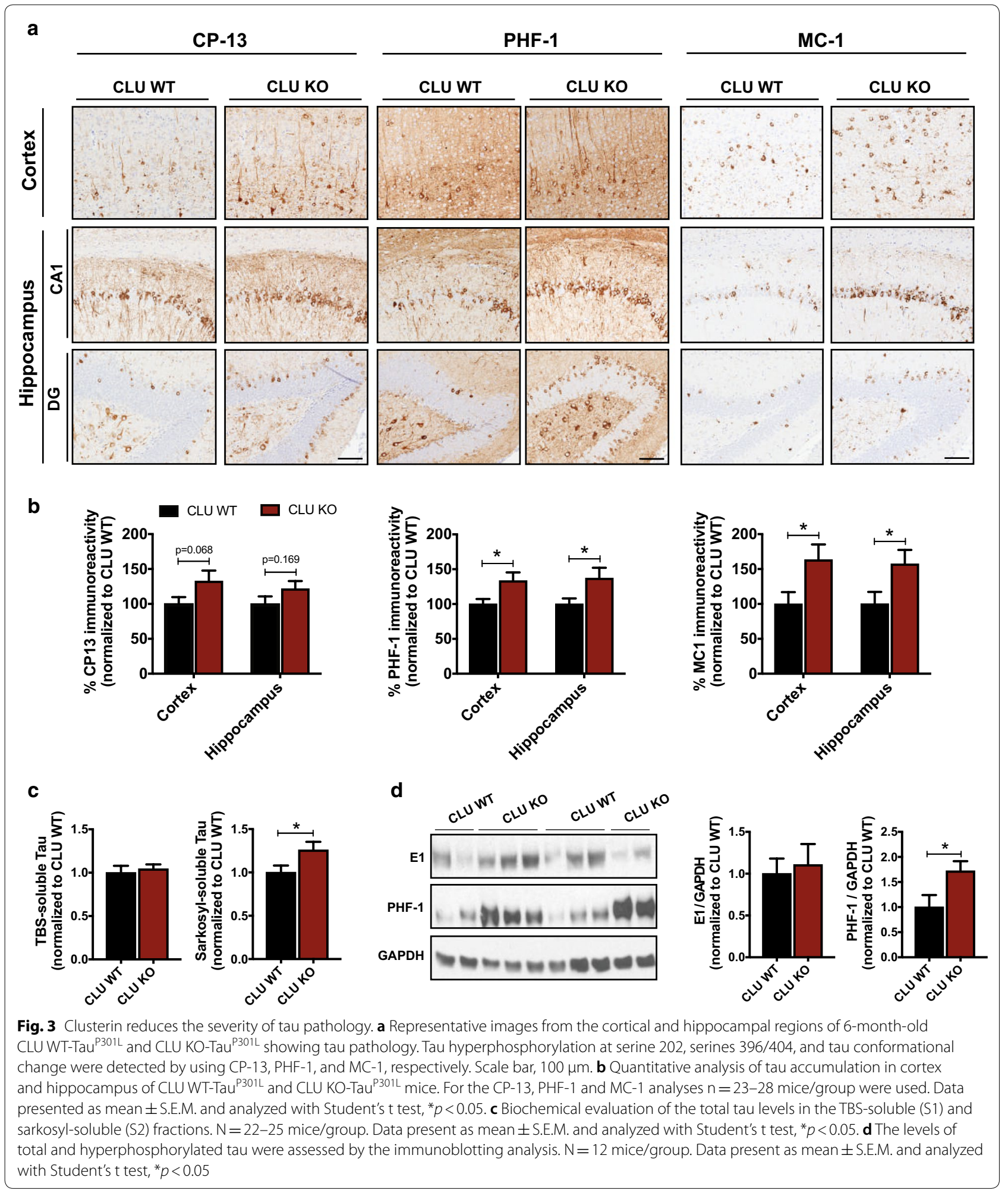

early stage of pathology, we also examined the degree of phosphorylation at the pS396 and pS404 sites that are thought to represent more mature tau deposits. Notably, the immunolabeling with PHF-1 showed significantly stronger immunoreactivity in the cortical $(* p<0.05)$ and hippocampal $(" p<0.05)$ regions of CLU KO-Tau ${ }^{\text {P301L }}$ 
mice compared to CLU WT-Tau ${ }^{\text {P301L }}$ animals (Fig. 3a, b). To further characterize the effect of CLU on tau pathology, we examined the abnormal conformational change of tau in these mice using MC-1 immunolabeling. Similar to the effects on PHF-1, CLU KO-Tau ${ }^{\mathrm{P} 301 \mathrm{~L}}$ mice exhibited significantly higher $\mathrm{MC}-1$ positive immunoreactivity in both cortex (" $p<0.05)$ and hippocampus (" $p<0.05)$ compared to CLU WT-Tau ${ }^{\text {P301L }}$ animals (Fig. 3a, b and Additional file 1: Fig. S3). Consistent with this, Gallyas silver stain, detecting mature tau fibrils, was more abundant in cortex of CLU KO-Tau ${ }^{\mathrm{P} 301 \mathrm{~L}}$ mice (" $\left.p<0.05\right)$; although, there was no significant difference in the Gallyas-positive deposits in the hippocampus between CLU WT-Tau ${ }^{\text {P301L }}$ and CLU KO-Tau ${ }^{\mathrm{P} 301 \mathrm{~L}}$ mice (Additional file 1: Fig. S4a, b). In addition, mice lacking CLU exhibited a significantly smaller area of the hippocampus compared to control animals, suggesting that augmented tau pathology in these animals was accompanied by hippocampal atrophy (Additional file 1: Fig. S4c).

The effect of CLU expression on tau pathology led us to evaluate the impact of CLU on tau solubility by assessing tau levels biochemically. To this end, we isolated different tau species from cortical tissue and evaluated total tau levels in TBS-soluble and sarkosyl-soluble fractions, using a highly sensitive meso-scale discovery (MSD) assay. As expected, tau levels in the soluble fraction, reflecting less aggregated forms of tau, were not significantly different between CLU genotypes (Fig. 3c). However, we observed significantly higher levels of oligomeric tau in the sarkosyl-soluble fraction in CLU KO-Tau ${ }^{\mathrm{P} 301 \mathrm{~L}}$ mice (" $p<0.05)$ compared to CLU WT-Tau ${ }^{\text {P301L }}$ animals (Fig. 3c). Immunobloting further showed a marked increase in the levels of phosphorylated tau $(" p<0.05)$ in cortex of CLU KO-Tau ${ }^{\mathrm{P} 301 \mathrm{~L}}$ mice compared to control CLU WT-Tau ${ }^{\mathrm{P} 301 \mathrm{~L}}$ animals, with no differences in the total tau levels observed between two groups (Fig. 3d). Taken together, these results indicate that the presence of CLU had a significant effect on tau pathology, particularly the more severe or mature forms of tau.

Previous studies have shown abundant gliosis associated with tau accumulation in the brain [22]. Thus, we performed a histological examination of astrogliosis using glial fibrillary acidic protein (GFAP) as a marker for reactive astrocytes. As expected, we observed marked astrogliosis in the cortex and hippocampus of mice injected with AAV-Tau ${ }^{\mathrm{P} 301 \mathrm{~L}}\left({ }^{* * * * * *} p<0.0001\right)$ compared to animals injected with AAV-GFP (Additional file 1: Fig. S5). Additionally, CLU genotype further influenced astrogliosis, with CLU KO-Tau ${ }^{\mathrm{P} 301 \mathrm{~L}}$ mice having higher levels of astrogliosis in cortex $(p=0.055)$, but not hippocampus $(p=0.58)$ compared to CLU WT-Tau ${ }^{\text {P301L }}$ animals (Additional file 1: Fig. S5). We also observed a significant increase in microgliosis associated with tau deposits $\left.{ }^{* * *} p<0.01\right)$, but we did not find differences between the CLU genotypes (Additional file 1: Fig. S5).

\section{CLU significantly inhibits tau fibril formation}

To investigate a possible mechanism by which CLU may impact severity and progression of tau pathology, we evaluated the ability of CLU to influence tau fibrillization in a cell-free system. To this end, we incubated recombinant human tau protein (4RON) with recombinant human CLU and assessed the effects over the course of $24 \mathrm{~h}$. Strikingly, we observed that the presence of CLU significantly prevented the assembly and aggregation of tau $\left.{ }^{(* * * * *} p<0.001\right)$ in this thioflavin T assay (Fig. 4a, b). Following tau fibrillization, we assessed CLU and tau levels in the remaining soluble and insoluble fractions using Western blot analysis. While the absence of CLU in the preparations led to the robust aggregation of tau in the insoluble fraction, the presence of CLU promoted tau solubility as detected by a higher amount of CLU and tau retained in the soluble fraction with only small amount of tau present as insoluble aggregates (Fig. 4c).

Importantly, a similar effect was observed when recombinant tau was incubated with tau paired helical filaments (PHFs) purified from AD brains, and with increasing CLU concentrations. The presence of CLU led to a significant reduction of the tau assembly measured by thioflavine $\mathrm{T}$ fluorescence (Fig. 4d).

\section{Discussion}

In the present study, we examined the functional relationship between CLU and tau using the AAV-based approach to deliver human tau bearing the P301L mutation into CLU WT and CLU KO animals. We found that loss of CLU was associated with the aggravated accumulation of pathological tau and behavioral abnormalities in our mouse model of tauopathy. We also found that CLU co-localized with tau deposits in human tauopathy cases and that direct interaction of CLU and tau in a cell-free system led to a dramatic reduction of the tau filament formation.

CLU is upregulated in AD cases and numerous genetic studies have found a strong association of the protective $T$ allele of the rs11165000 SNP in the CLU gene with higher CLU levels in AD patients [9, 13, 20], suggesting that the increased CLU expression may be beneficial. In addition, the link between elevated CLU and tau pathology has been recently reported in $\mathrm{AD}$ patients [21]. Consistent with these findings, we observed highly upregulated levels of CLU in AD patients compared to control individuals. Importantly, our study is the first to show a significant increase in CLU levels in primary tauopathies compared to controls, suggesting that CLU upregulation occurs not only in the presence of amyloid 

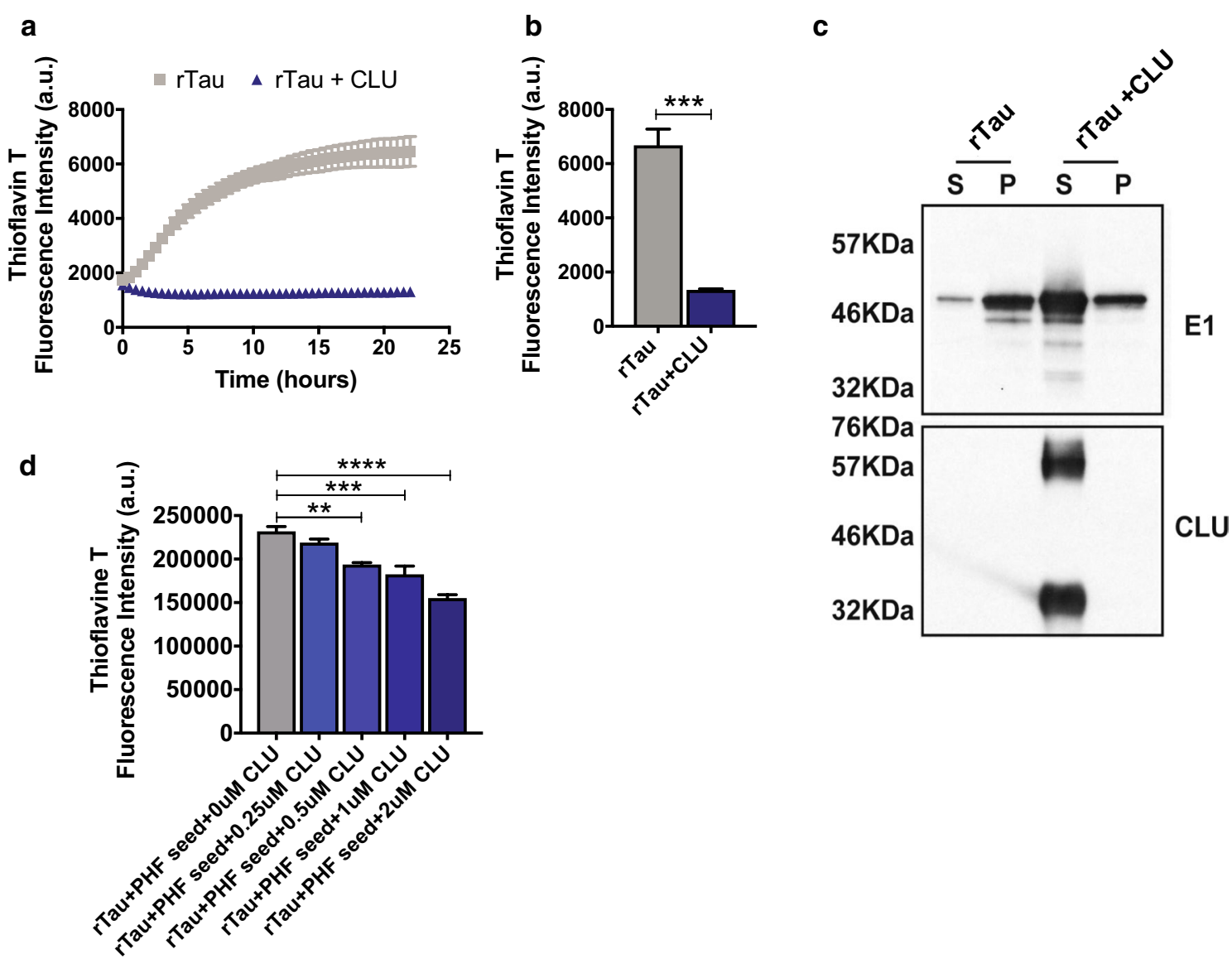

Fig. 4 CLU binds to tau and prevents tau aggregation. a In vitro tau fibrilization assay was performed with the 4 RON tau isoform in the presence or absence of recombinant human CLU and thioflavine T intensity was monitored during 24-h time course. $\mathbf{b}$ Quantification of thioflavine T signal at the 24-h time point. Four replicates were used. Data present as mean \pm S.E.M. and analyzed by Student's $t$ test ${ }^{* * *} p<0.001$. c Western blot analysis of soluble (supernatant, s) and insoluble (pellet, p) fractions following tau fibril assembly in the presence or absence of exogenous CLU. $\mathbf{d}$ Tau assembly assay performed with $4 R O N$ recombinant tau, tau PHFs from AD brains, and increasing concentrations of recombinant CLU. Four replicates were used. Data present as mean \pm S.E.M. and analyzed by one-way ANOVA, ${ }^{* *} p<0.01,{ }^{* * *} p<0.001,{ }^{* * * *} p<0.0001$

plaques but also tau pathology. As previously shown [6], we found an extensive CLU co-localization with amyloid deposits in AD brains. Moreover, CLU immunoreactivity was present in many NFT-bearing neurons in both primary and secondary tauopathies suggesting tau-specific changes in CLU expression.

Importantly, our study also showed that CLU significantly influenced the formation of insoluble tau filaments in vitro, thus elucidating a possible mechanism by which CLU may modulate tau aggregation. However, it remains unclear where the in vivo interaction between CLU and tau may occur. Although tau predominantly exists as an intracellular protein, recent studies have suggested that tau can be also released from neurons and undergo cell-to-cell transmission [23]. Given that CLU is mainly a secreted protein [5], the potential binding to tau may occur in the extracellular space, possibly influencing tau spreading. Previous studies have also suggested the existence of intracellular forms of CLU that would emerge from alternative splicing, translation-initiation events, or failed translocation. Leskov and colleagues have reported the expression of the nuclear form of CLU that locates to the nucleus in response to cellular stress [14]. Other intracellular CLU forms have also been proposed, including partially-glycosylated form of secreted CLU translocated to the cytosol or mitochondrial membrane, therefore escaping the secretory pathway [18]. A recent report has shown the interaction of intracellular CLU with the wild-type and P301L mutant tau protein in vitro, suggesting that CLU may also regulate tau accumulation by its binding inside neurons [26].

Two recent studies have centered on defining the role of another abundantly expressed apolipoprotein in the brain and a major AD risk gene, apolipoprotein $\mathrm{E}$ (ApoE), in tau pathology [22, 25]. Shi and colleagues have shown that $A P O E \& 4$ induced a more severe disease phenotype 
with augmented neurodegeneration and neuroinflammation compared to $A P O E \varepsilon 3$ and $A P O E \varepsilon 2$ genotypes in P301S transgenic mice [22]. Another study led by Zhao and colleagues has reported a strong link between $A P O E$ $\varepsilon 2$ and increased pathological changes of tau in the brains of mice injected with AAV-Tau ${ }^{\mathrm{P} 01 \mathrm{~L}}$ [25]. Moreover, the authors have found a significant association between the $A P O E \varepsilon 2 / \varepsilon 2$ genotype and increased risk of progressive supranuclear palsy [25]. Although these two seminal reports have demonstrated the associations of exacerbated tau pathology with different APOE isoforms, an effect possibly attributable to different model systems, nonetheless they provided new insights into APOE function in AD. It remains to be elucidated whether CLU and apoE impact the tau accumulation through the same mechanism in human tauopathies.

Although the effect of CLU on amyloid pathology has been well established, our findings suggest that CLU also plays a significant role in modulating tau pathology, thus providing new insights into CLU contribution to AD pathophysiology. Future studies into detailed mechanisms underlying the relationship of CLU with tau, perhaps in concert with apoE, may advance our understanding of $\mathrm{AD}$ and other tauopathies.

\section{Supplementary information}

Supplementary information accompanies this paper at https://doi. org/10.1186/s40478-020-01079-1.

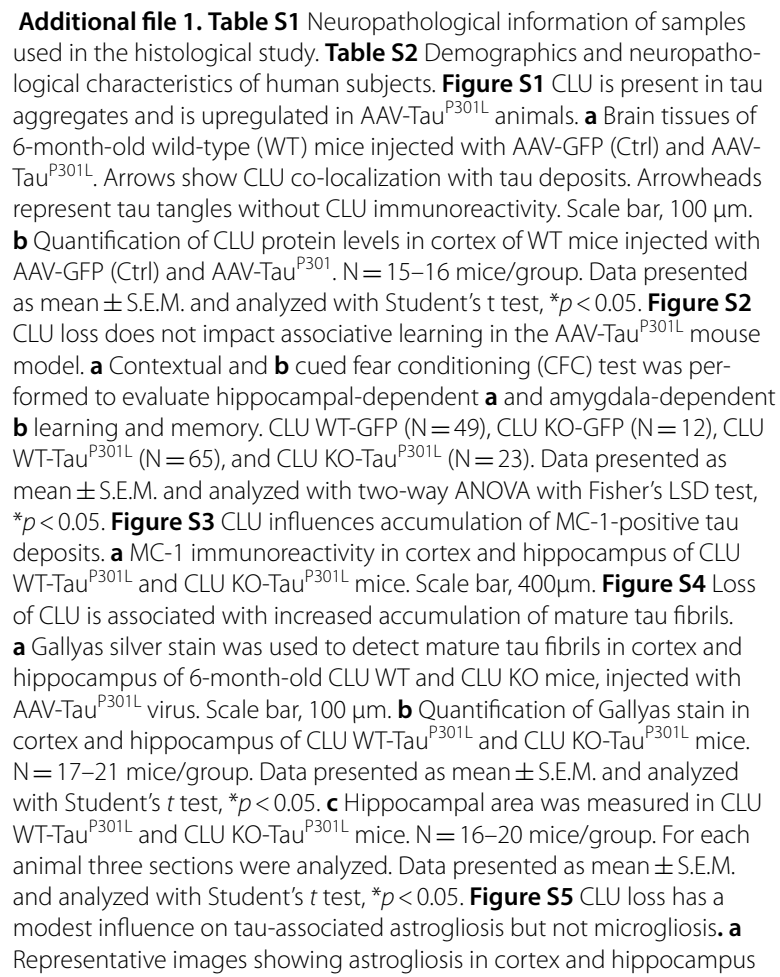

of 6-month-old CLU WT and CLU KO mice injected with AAV-GFP or AAV-Tau ${ }^{\text {P301L }}$. Scale bar, $100 \mu \mathrm{m}$. b Quantitative analysis of GFAP immunoreactivity in cortex and hippocampus. AAV-GFP N =6-8 mice/group, AAV-Tau ${ }^{\text {P301L }} \mathrm{N}=22-24$ mice/group. Data present as mean \pm S.E.M. and analyzed with two-way ANOVA followed by Tukey correction for multiple comparisons, ${ }^{*} p<0.05,{ }^{* *} p<0.01$. c Microgliosis was evaluated by IBA1 immunoreactivity in cortex and hippocampus of CLU WT and CLU KO mice injected with AAV-GFP or AAV-TauP301L. Scale bar, $100 \mu \mathrm{m}$. d Quantification of IBA1 immunoreactivity in cortex and hippocampus. AAV-GFP $\mathrm{n}=6-8$ mice/group, AAV-Tau ${ }^{\text {P301L }} \mathrm{N}=22-24$ mice/group. Data present as mean \pm S.E.M. and analyzed with two-way ANOVA followed by Tukey correction for multiple comparisons.

\section{Abbreviations}

AAV: Adeno-associated viral vectors; AD: Alzheimer's disease; APOE: Apolipoprotein E; CBD: Corticobasal degeneration; CLU: Clusterin; ELISA: Enzymelinked immunosorbent assay; GFAP: Glial fibrillary acidic protein; GFP: Green fluorescent protein; GWAS: Genome-wide association studies; IBA1: Ionized calcium binding adaptor molecule 1; MSD: Meso Scale Discovery; PiD: Pick's disease; PSP: Progressive supranuclear palsy; SNP: Single nucleotide polymorphism; TBS: Tris-buffered saline.

\section{Acknowledgements}

We appreciate the generosity of patients and their families for their generous donation of brain samples for this study. We would like to thank Dr. Peter Davies for providing the CP-13, PHF-1, and MC-1 antibodies. We thank all other members of the Fryer lab for helpful discussions and technical advice.

\section{Authors' contribution}

JDF contributed to the conception and design of the project. JDF and AMW analyzed the data and wrote the manuscript. AMW, YC, JPS, SSK, CNC, MY, TDJ, KEB, TJB, AK, AA performed experiments. DWD, MD, CHFDC provided the human specimens. VRP, MCC, ALL processed tissue for histological analysis. LD, KJW, LP produced AAV viral vectors.

\section{Funding}

J.D.F. was supported by the Mayo Foundation, the Mayo Clinic Center for Individualized Medicine, a Mayo Clinic Gerstner Family Career Development Award, the Ed and Ethel Moore Alzheimer's Disease Research Program of Florida Department of Health (6AZ06), the Gilmer Family Foundation, Alzheimer's Association NIRP-12-25928, The Rotary Coins for Alzheimer's Research Trust Fund, CureAlz Foundation, Ben-Dov Family Foundation, and NIH Grants NS084974, AG062556, AG0621 10, NS094137, AG057997, AG062077, NS1 10435, AG047327, and AG049992.

\section{Availability of data and materials}

All data used and analyzed for the current study are available from the corresponding author on reasonable request.

\section{Ethics approval and consent to participate}

Not applicable.

\section{Consent for publication}

All authors have given their consent for publication.

\section{Competing interests}

The authors declare that no competing interests exist.

\section{Author details}

${ }^{1}$ Department of Neuroscience, Mayo Clinic, Collaborative Research Building CR03-010 13400 E. Shea Blvd, Scottsdale, AZ 85259, USA. ${ }^{2}$ Department of Neuroscience, Mayo Clinic, Jacksonville, FL 32224, USA. ${ }^{3}$ Neuroscience Graduate Program, Mayo Clinic Graduate School of Biomedical Sciences, Scottsdale, AZ 85259, USA.

Received: 9 November 2020 Accepted: 11 November 2020

Published online: 01 December 2020 


\section{References}

1. Arriagada PV, Growdon JH, Hedley-Whyte ET, Hyman BT (1992) Neurofibrillary tangles but not senile plaques parallel duration and severity of Alzheimer's disease. Neurology 42:631-639. https://doi.org/10.1212/ wnl.42.3.631

2. Bell RD, Sagare AP, Friedman AE, Bedi GS, Holtzman DM, Deane R, Zlokovic BV (2007) Transport pathways for clearance of human Alzheimer's amyloid beta-peptide and apolipoproteins $\mathrm{E}$ and $\mathrm{J}$ in the mouse central nervous system. J Cereb Blood Flow Metab 27:909-918. https://doi. org/10.1038/sj.jcbfm.9600419

3. Cook C, Kang SS, Carlomagno Y, Lin WL, Yue M, Kurti A, Shinohara M, Jansen-West K, Perkerson E, Castanedes-Casey M et al (2015) Tau deposition drives neuropathological, inflammatory and behavioral abnormalities independently of neuronal loss in a novel mouse model. Hum Mol Genet 24:6198-6212. https://doi.org/10.1093/hmg/ddv336

4. DeMattos RB, O'Dell MA, Parsadanian M, Taylor JW, Harmony JA, Bales KR, Paul SM, Aronow BJ, Holtzman DM (2002) Clusterin promotes amyloid plaque formation and is critical for neuritic toxicity in a mouse model of Alzheimer's disease. Proc Natl Acad Sci USA 99:10843-10848. https://doi. org/10.1073/pnas.162228299

5. Foster EM, Dangla-Valls A, Lovestone S, Ribe EM, Buckley NJ (2019) Clusterin in Alzheimer's disease: mechanisms, genetics, and lessons from other pathologies. Front Neurosci 13:164. https://doi.org/10.3389/fnins 2019.00164

6. Giannakopoulos P, Kovari E, French LE, Viard I, Hof PR, Bouras C (1998) Possible neuroprotective role of clusterin in Alzheimer's disease: a quantitative immunocytochemical study. Acta Neuropathol 95:387-394. https ://doi.org/10.1007/s004010050815

7. Glenner GG, Wong CW (1984) Alzheimer's disease: initial report of the purification and characterization of a novel cerebrovascular amyloid protein. Biochem Biophys Res Commun 120:885-890. https://doi. org/10.1016/s0006-291x(84)80190-4

8. Grundke-labal I, labal K, Quinlan M, Tung YC, Zaidi MS, Wisniewski HM (1986) Microtubule-associated protein tau. A component of Alzheimer paired helical filaments. J Biol Chem 261:6084-6089

9. Harold D, Abraham R, Hollingworth P, Sims R, Gerrish A, Hamshere ML, Pahwa JS, Moskvina V, Dowzell K, Williams A et al (2009) Genome-wide association study identifies variants at CLU and PICALM associated with Alzheimer's disease. Nat Genet 41:1088-1093. https://doi.org/10.1038/ ng. 440

10. Janocko NJ, Brodersen KA, Soto-Ortolaza Al, Ross OA, Liesinger AM, Duara R, Graff-Radford NR, Dickson DW, Murray ME (2012) Neuropathologically defined subtypes of Alzheimer's disease differ significantly from neurofibrillary tangle-predominant dementia. Acta Neuropathol 124:681-692. https://doi.org/10.1007/s00401-012-1044-y

11. Josephs KA, Whitwell JL, Ahmed Z, Shiung MM, Weigand SD, Knopman DS, Boeve BF, Parisi JE, Petersen RC, Dickson DW et al (2008) Betaamyloid burden is not associated with rates of brain atrophy. Ann Neurol 63:204-212. https://doi.org/10.1002/ana.21223

12. Kovacs GG (2015) Invited review: neuropathology of tauopathies: principles and practice. Neuropathol Appl Neurobiol 41:3-23. https://doi. org/10.1111/nan.12208

13. Lambert JC, Heath S, Even G, Campion D, Sleegers K, Hiltunen M, Combarros O, Zelenika D, Bullido MJ, Tavernier B et al (2009) Genome-wide association study identifies variants at CLU and CR1 associated with Alzheimer's disease. Nat Genet 41:1094-1099. https://doi.org/10.1038/ ng.439

14. Leskov KS, Klokov DY, Li J, Kinsella TJ, Boothman DA (2003) Synthesis and functional analyses of nuclear clusterin, a cell death protein. J Biol Chem 278:11590-11600. https://doi.org/10.1074/jbc.M209233200
15. May PC, Johnson SA, Poirier J, Lampert-Etchells M, Finch CE (1989) Altered gene expression in Alzheimer's disease brain tissue. Can J Neurol Sci 16:473-476. https://doi.org/10.1017/s0317167100029796

16. Nilselid AM, Davidsson P, Nagga K, Andreasen N, Fredman P, Blennow K (2006) Clusterin in cerebrospinal fluid: analysis of carbohydrates and quantification of native and glycosylated forms. Neurochem Int 48:718-728. https://doi.org/10.1016/j.neuint.2005.12.005

17. Oda T, Wals P, Osterburg HH, Johnson SA, Pasinetti GM, Morgan TE, Rozovsky I, Stine WB, Snyder SW, Holzman TF et al (1995) Clusterin (apoJ) alters the aggregation of amyloid beta-peptide (A beta 1-42) and forms slowly sedimenting A beta complexes that cause oxidative stress. Exp Neurol 136:22-31. https://doi.org/10.1006/exnr.1995.1080

18. Rohne P, Prochnow H, Koch-Brandt C (2016) The CLU-files: disentanglement of a mystery. Biomol Concepts 7:1-15. https://doi.org/10.1515/ bmc-2015-0026

19. Schrijvers EM, Koudstaal PJ, Hofman A, Breteler MM (2011) Plasma clusterin and the risk of Alzheimer disease. JAMA 305:1322-1326. https://doi. org/10.1001/jama.2011.381

20. Schurmann B, Wiese B, Bickel H, Weyerer S, Riedel-Heller SG, Pentzek M, Bachmann C, Williams J, van den Bussche H, Maier W et al (2011) Association of the Alzheimer's disease clusterin risk allele with plasma clusterin concentration. J Alzheimers Dis 25:421-424. https://doi.org/10.3233/JAD2011-110251

21. Shepherd CE, Affleck AJ, Bahar AY, Carew-Jones F, Halliday GM (2020) Intracellular and secreted forms of clusterin are elevated early in Alzheimer's disease and associate with both Abeta and tau pathology. Neurobiol Aging 89:129-131. https://doi.org/10.1016/j.neurobiola ging.2019.10.025

22. Shi Y, Yamada K, Liddelow SA, Smith ST, Zhao L, Luo W, Tsai RM, Spina S, Grinberg LT, Rojas JC et al (2017) ApoE4 markedly exacerbates taumediated neurodegeneration in a mouse model of tauopathy. Nature 549:523-527. https://doi.org/10.1038/nature24016

23. Wang Y, Mandelkow E (2016) Tau in physiology and pathology. Nat Rev Neurosci 17:5-21. https://doi.org/10.1038/nrn.2015.1

24. Wojtas AM, Kang SS, Olley BM, Gatherer M, Shinohara M, Lozano PA, Liu CC, Kurti A, Baker KE, Dickson DW et al (2017) Loss of clusterin shifts amyloid deposition to the cerebrovasculature via disruption of perivascular drainage pathways. Proc Natl Acad Sci USA 114:E6962-E6971. https://doi. org/10.1073/pnas.1701137114

25. Zhao N, Liu CC, Van Ingelgom AJ, Linares C, Kurti A, Knight JA, Heckman MG, Diehl NN, Shinohara M, Martens YA et al (2018) APOE epsilon2 is associated with increased tau pathology in primary tauopathy. Nat Commun 9:4388. https://doi.org/10.1038/s41467-018-06783-0

26. Zhou Y, Hayashi I, Wong J, Tugusheva K, Renger JJ, Zerbinatti C (2014) Intracellular clusterin interacts with brain isoforms of the bridging integrator 1 and with the microtubule-associated protein Tau in Alzheimer's disease. PLoS ONE 9:e103187. https://doi.org/10.1371/journal.pone.01031 87

27. Zlokovic BV, Martel CL, Matsubara E, McComb JG, Zheng G, McCluskey RT, Frangione B, Ghiso J (1996) Glycoprotein 330/megalin: probable role in receptor-mediated transport of apolipoprotein $\mathrm{J}$ alone and in a complex with Alzheimer disease amyloid beta at the blood-brain and bloodcerebrospinal fluid barriers. Proc Natl Acad Sci U S A 93:4229-4234. https ://doi.org/10.1073/pnas.93.9.4229

\section{Publisher's Note}

Springer Nature remains neutral with regard to jurisdictional claims in published maps and institutional affiliations. 